\title{
In der Menopause ist Hopfen und Malz nicht verloren
}

Die Hormonsubstitutionstherapie in der Menopause ist in Verruf geraten. Ein Hopfenextrakt könnte eine wirksame Alternative sein.

- Ein internationales Team rekrutierte 396 Frauen mit menopausalen Beschwerden für eine placebokontrollierte doppelblinde Crossover-Studie. Die Patientinnen erhielten jeweils acht Wochen lang entweder einen Hopfenextrakt, der auf $100 \mu \mathrm{g} 8$ 8-Prenylnaringenin/Tag standardisiert worden war, oder Placebo. Der Therapieerfolg wurde mit validierten Symptomscores quantifiziert.

Die Ergebnisse zeigen, dass unter Hopfenmedikation die menopausalen Beschwerden signifikant deutlicher rückläu- fig waren als unter Placebo. Die Autoren meinen daher, dass der Extrakt „eine interessante Alternative“ für dieses Patientengut darstellt.

\section{Kommentar}

Diese Studie scheint gut gemacht zu sein, und ihre Ergebnisse sind daher wohl auch zuverlässig. Die Autoren führen die klinischen Effekte auf den Phytoöstrogengehalt des Hopfens zurück. Ob Hopfen tatsächlich eine echte Alternative zur Hormonsubstituion darstellt, mag bezweifelt werden. Die Effektgröße scheint beim Hopfen geringer zu sein. Hinzu kommt, dass seine Wirksamkeit längst nicht so gut belegt ist. Am wichtigsten erscheint mir jedoch die Frage der Nebenwirkungen zu sein. Hormone sind in Verruf gekommen, als Studien mit sehr großen Fallzahlen relativ geringe Risiken aufzeigten. Zu Hopfen und ähnlichen Phytoöstrogen-Präparaten haben wir keine auch nur annährend ähnlich umfangreiche Daten. Wir wissen daher nicht, ob sie mit mehr oder weniger Risiken als die Hormontherapie belastet sind.

E. ERNST =

A randomized, double-blind, placebo-controlled, cross-over pilot study on the use of a standardized hop extract to alleviate menopausal discomforts. Phytomedicine 17 (2010) 389-396 